\title{
Influence of Lower Extremity Strength of Healthy Older Adults on the Outcome of an Induced Trip
}

\author{
Michael J. Pavol, PhD, ${ }^{*+}$ Tammy M. Owings, MS, * Kevin T. Foley, MD, \\ and Mark D. Grabiner, PhD*
}

OBJECTIVES: To determine whether decreased lower extremity strength contributes to trip-related falls in older adults.

DESIGN: A cross-sectional sample of older adults were safety-harnessed and tripped while walking using a concealed, mechanical obstacle. Lower extremity strength was compared between trip outcome groups.

SETTING: A biomechanics research laboratory.

PARTICIPANTS: Seventy-nine healthy, community-dwelling adults aged 65 and older (50 women).

MEASUREMENTS: Ankle, knee, and hip flexion and extension strength were measured isometrically and isokinetically. Measured strengths were subjected to a factor analysis. Strength factor scores were compared between those who recovered from the trip and those who fell by three previously identified mechanisms: during-step, after-step, and elevating-response falls.

RESULTS: Seven common factors, one associated with each direction of exertion at each joint and one with the time rate of moment increase, explained $88 \%$ of the variance in measured strength. The during-step $(n=5)$ fallers were significantly stronger in the ankle extension (plantarflexion), knee flexion, overall extension, and total strength factors than those who successfully recovered using a similar, lowering strategy $(\mathrm{n}=26)$. The elevating-response faller $(n=1)$ was stronger in the plantarflexion and overall extension factors than most of those who recovered using a similar, elevating strategy $(\mathrm{n}=11)$. Two of three after-step fallers were among the weakest subjects tested.

CONCLUSION: Weak older adults and the strongest older adults may be at greater risk of falling from a trip,

From the *Department of Biomedical Engineering, Lerner Research Institute, The Cleveland Clinic Foundation, Cleveland, Ohio; ${ }^{\dagger}$ Department of Physical Therapy, University of Illinois at Chicago, Chicago, Illinois; and ₹Section of Geriatric Medicine, The Cleveland Clinic Foundation, Cleveland, Ohio.

Funded by NIH-R01AG10557 (to MDG).

Presented at the 24th Annual Meeting of the American Society of Biomechanics, University of Illinois at Chicago, Chicago, Illinois, 2000.

Address correspondence to Michael J. Pavol, PhD, Department of Physical Therapy (MC898), University of Illinois at Chicago, 1919 West Taylor Street, 4th floor, Chicago, Illinois 60612. E-mail: mpavol@uic.edu although by different mechanisms. High strength may increase the likelihood of a during-step or elevating-response fall; decreased strength may increase the likelihood of an after-step fall. J Am Geriatr Soc 50:256-262, 2002.

Key words: older adults; strength; falling

A ging is associated with increased falling. Approximately one-third of community-dwelling older adults fall each year, and $11 \%$ of these falls result in serious injury. ${ }^{1-3}$ Aging is also associated with decreased lower extremity strength. Ankle, knee, and hip strength decline by up to $3 \%$ per year beyond the fifth decade of life, ${ }^{4-8}$ accompanied by a slowing of the rate of force generation. ${ }^{9,10}$ These strength declines could impair the ability to respond quickly and forcefully to prevent a fall after a postural disturbance, leading directly to increased falling by older adults.

In general, older adults who fall are significantly weaker than those who do not, both within community-dwelling and institutionalized populations. ${ }^{11-14}$ However, a causal relationship between weakness and the inability to recover after a large postural perturbation has not been demonstrated. Indeed, resistance training has not been specifically found to reduce fall incidence. ${ }^{15}$ Biomechanical analyses relating mechanisms of falling to measured strength are needed to establish the degree to which diminished strength contributes to falls by older adults. Importantly, such analyses may take into consideration that the strength demands of recovery, hence the influences of diminished strength, may vary with the perturbation type, the recovery strategy employed, and the mechanism of falling.

Of particular concern are falls due to tripping, which account for up to $53 \%$ of falls by older adults. ${ }^{1}$ We induced trips during gait in a population of healthy, community-dwelling older adults ${ }^{16}$ and found three distinct mechanisms of falling. ${ }^{17}$ Two mechanisms were associated with a lowering strategy response to the trip, in which the tripped foot was lowered on the near side of the obstacle, followed by a contralateral recovery step. "During-step" falls were associated with a faster walking speed at the time of the trip 
and a delayed lowering response, resulting in a rapid forward body rotation to the point of "falling" by recovery step ground contact. "After-step" falls were associated with a more anterior head-arms-torso center of mass at the time of the trip, excessive posttrip lumbar flexion, and buckling of the recovery limb after step ground contact. These latter two conditions led to a "fall" shortly after ground contact of the subsequent step. The third mechanism of falling was associated with an elevating strategy response, in which the tripped foot was immediately lifted across the obstacle. The "elevating-response" fall was associated with a faster walking speed and excessive posttrip lumbar flexion, with a progressive forward trunk rotation and hip descent to the point of "falling" after several subsequent steps. This study investigated whether decreased lower extremity strength may have contributed to these falls.

Diminished flexion or extension strength at the ankle, knee, or hip might reasonably contribute to the inability to recover. An initial lowering response of the tripped limb relies on increased knee flexion and hip extension moments. ${ }^{18}$ Recovery steps are effected through increased ankle flexion (dorsiflexion) and knee and hip flexion and extension moments in the stepping limb, ${ }^{18-20}$ likely accompanied by increased contralateral extension moments. Large knee and hip extension moments might be required to limit limb buckling and trunk flexion after recovery step ground contact. Finally, recovery may depend less on strength than on the ability to generate moments rapidly. ${ }^{21}$

Because recovery after a trip is a dynamic task that involves rapidly varying concentric and eccentric joint moments, measures used to investigate the role of strength in falling should appropriately characterize the dynamic functional strength capacity of older adults. Presently, there is little basis for selecting such measures; relationships between measured strengths for different joints, exertion directions, and joint velocities are not well established for older adults. Factor analysis provides one means of establishing an appropriate set of measures. Factor analysis uses patterns of correlation to identify the underlying factors that largely determine the values of a set of measured variables. A factor score, computed through a weighted sum of the measured variables, indicates the extent to which an individual possesses a factor.

To pursue our main objective, we needed to identify a set of factors that characterized the functional ankle, knee, and hip flexion and extension strength capacities of our previously-referenced population of healthy, communitydwelling older adults. ${ }^{16}$ Based on these "appropriate" measures of strength, our main objective was to test the hypothesis that, for each identified mechanism of falling, those who fell after the induced trip were weaker than those who successfully recovered using a similar recovery strategy.

\section{METHODS}

\section{Subjects}

Fifty women and 29 men (mean age \pm standard deviation $=$ $72 \pm 5$ years; height $=1.64 \pm 0.09$ meters; mass $=76.0 \pm$ $14.0 \mathrm{~kg}$ ), all healthy, community dwelling, and aged 65 and older, provided written informed consent and were paid to participate. A geriatrician screened subjects for ex- clusionary factors, including neurological, musculoskeletal, cardiovascular, and cognitive disorders and a history of repeated falling. A minimum bone mineral density of $0.65 \mathrm{~g} \cdot \mathrm{cm}^{-2}$, assessed by duel energy x-ray absoptiometry (Hologic QDR 1000, Waltham, MA), was required at the femoral neck. This experiment was part of a larger study of falling in these older adults. The Institutional Review Board of the Cleveland Clinic Foundation approved all protocols.

\section{Strength Measurement}

Isometric and isokinetic flexion and extension strength were measured at the right and left ankle, knee, and hip joints (KIN-COM $500 \mathrm{H}$, Chattanooga Corp., Chattanooga, TN). The exceptions were knee flexion and hip flexion strength. To reduce potential fatigue effects, these were measured only for the subject's preferred stepping limb, under the assumption that limb withdrawal strength was least likely to limit recovery. Ankle, knee, and hip joints were tested a week apart.

Isometric strength was measured through a single 3 -second maximum voluntary exertion (MVE) at each of a specified set of joint angles. Ankle strengths were tested at $-5^{\circ}, 10^{\circ}$, and $25^{\circ}$ of ankle extension (plantarflexion), knee flexion strength at $20^{\circ}, 40^{\circ}, 65^{\circ}$, and $90^{\circ}$ of flexion, knee extension strength at $20^{\circ}, 45^{\circ}, 70^{\circ}$, and $90^{\circ}$ of flexion, and hip strengths at $10^{\circ}, 25^{\circ}, 45^{\circ}$, and $60^{\circ}$ of flexion. Zero corresponded to a joint's orientation in the anatomical position. Isokinetic strength was measured concentrically and eccentrically at $30 \%$ second over the same, joint-specific range of motion. At this speed, the time rate of moment generation would minimally influence the strength measures. Tested ranges of motion were $-5^{\circ}$ to $35^{\circ}, 20^{\circ}$ to $90^{\circ}$, and $10^{\circ}$ to $60^{\circ}$ at the ankle, knee, and hip, respectively. Two MVE trials were performed for each isokinetic condition. Movement thresholds exceeded the passive force by 22 Newtons in dorsiflexion, 45 Newtons otherwise. Subjects were instructed to push or pull "as hard and fast as you can" at the start of and throughout each MVE. Arms were kept folded across the chest. Verbal encouragement was provided. At least 1 minute of rest separated MVEs.

Exertions were blocked by limb, exertion direction, and then exertion type. Limbs and exertion directions were tested in random order. Isometric, concentric, and eccentric exertions were tested in this order. Orders of the isometric joint angles were randomized. Warm-up exertions preceded testing in each direction and practice MVEs preceded each exertion type.

Passive joint moment data were measured during three passive trials of isokinetic flexion-extension at $30 \%$ second after the strength measurements for each limb. Subjects neither aided nor resisted the dynamometer-imposed motion. The passive range of motion exceeded that of the isokinetic strength measurements by $5^{\circ}$ at each endpoint. Practice trials were performed until obtaining repeatable force-angle relationships. Trials visually judged as inconsistent were repeated.

Ankle and hip strength were measured in the supine position. Knee strength was measured in a seated position. Subjects were secured to the dynamometer at the foot, distal leg, and distal thigh for ankle, knee, and hip strength measurements, respectively. Restraint straps across the distal thigh, waist, and chest were employed, as appropri- 
ate, to minimize movement proximal to the tested joint. The ipsilateral knee was supported in slight flexion for ankle strength measurement. Because ipsilateral knee motion was not regulated during hip strength measurement, an electrogoniometer measured the knee angle over each hip MVE. The electrogoniometer angle and the force, angle, and velocity signals from the dynamometer were recorded at $500 \mathrm{~Hz}$.

\section{Induced Trip Protocol}

In a separate, final session, subjects were placed in a safety harness and tripped during self-selected "normal" gait. ${ }^{16}$ Subjects wore a full-body safety harness attached to a ceiling-mounted track by a pair of shock-absorbing dynamic ropes, typically used for fall protection in rock climbing. Rope lengths were adjusted so the wrists and knees could not touch the floor. A load cell (Omega Engineering, Stamford, CT) measured the force exerted on the ropes by the subject.

Trips were induced using a concealed, pneumatically driven, metal obstacle. This obstacle would rise 5.1 centimeters from the floor when manually triggered by the investigator, inducing a trip by obstructing the toe of the shoe of the swing foot during mid-to-late swing. A decoy "tripping rope," placed across the gait path 1.5 meters before the mechanical obstacle, implicitly misled the subject as to the time, location, and mechanism of the trip.

Subjects were informed that a trip would take place during an upcoming, but unspecified, trial. Instructions were to walk at a self-selected "normal" speed from a designated starting point to a designated stopping point, looking straight ahead. If tripped, subjects were to recover and continue walking. Only one attempt was made to trip each subject.

\section{Strength Data Analysis}

Strength was quantified, for each direction of exertion at each joint, by measures of isometric and isokinetic active moment capacity and by the maximum time rate of moment increase. Joint moments were computed from the dynamometer-measured forces, using a subject-specific, validated, mathematical model to compensate for artifacts introduced by gravity, system accelerations and compli- ances, distal limb segment motion, and off-axis force components. ${ }^{22}$ Moments were low-pass filtered at $10 \mathrm{~Hz}, 8 \mathrm{~Hz}$, and $6 \mathrm{~Hz}$ at the ankle, knee, and hip, respectively, using recursive fourth-order Butterworth filters.

Active moments, representing voluntary strength, were obtained from the computed moments by subtracting the corresponding angle-, direction-, and limb-specific passive moment. Passive moments were averaged across trials, with isometric passive moments determined by averaging across directions. In computing active hip moments, passive moments were linearly adjusted according to the deviation of the knee angle from that in the averaged passive trials, using relationships determined from the pooled passive data of all subjects.

Isometric strength for a given direction and joint was derived from the bilateral-maximum active moments at each tested joint angle. MVEs in which the initial baseline moment was inconsistent with the passive moment were excluded, as were hip MVEs in which the knee angle (i.e., biarticular muscle length) at the maximum moment differed substantially from that of the passive trials. For each bilateral-maximum active moment, the moment capacity at a reference joint angle (Table 1) was estimated by scaling the magnitude of a standardized moment-angle relationship to include the observed moment. The average of the two largest estimates of moment capacity at the reference angle represented isometric active moment capacity.

The standardized moment-angle relationships were polynomial functions of joint angle, specific to the exertion direction and joint. ${ }^{23}$ Relationships were quadratic at the ankle, cubic at the knee and hip. These were derived, using nonlinear regression, from the pooled data of all subjects with valid data at all tested angles. Reference joint angles are generally associated with the largest moments within the tested range of motion.

Concentric and eccentric isokinetic strengths were quantified by the corresponding bilateral-maximum active moments at the midpoint of the tested range of motion. Data for a given isokinetic condition were discarded if all corresponding exertions appeared submaximal, based on an inappropriate location of the peak active moment within the tested range of motion. ${ }^{23,24}$ Hip strength trials were discarded if a large-amplitude moment oscillation oc-

Table 1. Lower Extremity Strength in the Sample Population of Older Adults $(n=63)$

\begin{tabular}{|c|c|c|c|c|c|c|}
\hline \multirow[b]{2}{*}{$\begin{array}{l}\text { Exertion } \\
\text { Direction }\end{array}$} & \multicolumn{2}{|c|}{ Isometric } & \multicolumn{3}{|c|}{$30 \%$ s Isokinetic } & \multirow[b]{2}{*}{$\begin{array}{c}\text { Maximum Time Rate } \\
\text { of Increase }(\% b w \cdot b h / s) \\
\text { mean } \pm S D(r)^{\dagger}\end{array}$} \\
\hline & $\begin{array}{c}\text { Strength } \\
(\% \text { bw·bh) } \\
\text { mean } \pm \text { SD }\end{array}$ & $\begin{array}{c}\text { Reference } \\
\text { Joint } \\
\text { Angle }\end{array}$ & $\begin{array}{c}\text { Concentric (\%bw·bh) } \\
\text { mean } \pm \mathrm{SD}(r)^{\dagger}\end{array}$ & $\begin{array}{c}\text { Eccentric }(\% b w \cdot b h) \\
\text { mean } \pm S D(r)^{\dagger}\end{array}$ & $\begin{array}{l}\text { Reference } \\
\text { Joint } \\
\text { Angle }\end{array}$ & \\
\hline Dorsiflexion & $2.2 \pm 0.6$ & $18^{\circ *}$ & $1.4 \pm 0.4(.85)$ & $2.2 \pm 0.5(.81)$ & $15^{\circ}$ & $8.7 \pm 2.7(.73)$ \\
\hline Knee extension & $12.2 \pm 2.5$ & $67^{\circ *}$ & $9.2 \pm 2.5(.80)$ & $13.3 \pm 3.2(.76)$ & $55^{\circ}$ & $33.8 \pm 13.4(.77)$ \\
\hline Hip flexion & $9.8 \pm 2.3$ & $0^{\circ}$ & $6.2 \pm 1.5(.82)$ & $8.1 \pm 1.9(.81)$ & $35^{\circ}$ & $35.8 \pm 14.0(.63)$ \\
\hline Hip extension & $8.3 \pm 2.6$ & $60^{\circ}$ & $6.0 \pm 2.3(.82)$ & $8.2 \pm 2.3(.79)$ & $35^{\circ}$ & $21.7 \pm 9.4(.67)$ \\
\hline
\end{tabular}

${ }^{\dagger} \mathbf{r}=$ Pearson correlation to the corresponding isometric active moment capacity (i.e., strength).

* Corresponds to the peak moment in the derived moment-angle relationship.

$\%$ bw $\cdot$ bh $=$ percentage of body weight times body height; SD $=$ standard deviation. 
curred across the midrange angle or if the knee angle at midrange differed substantially from that of the passive trials. In total, $14 \%$ of all trials were excluded or discarded as possibly misrepresentative of actual strength.

The maximum time rate of moment increase was determined across all exertions in a given direction based on the moving average of the time rate of change of the moment over a period of 100 milliseconds at the ankle and knee, 150 milliseconds at the hip. A maximum rate was extracted from each trial, and the two largest extracted rates were averaged.

All strength measures were normalized to a percentage of body weight times body height $(\% \mathrm{bw} \cdot \mathrm{bh})$ using allometric scaling. ${ }^{25}$

\section{Trip Outcome Classification}

Each trip outcome was classified as a recovery, fall, rope assist, or miss. ${ }^{16}$ Falls occurred if the subject was fully supported by the safety harness. Recoveries occurred if an average force of less than $5 \%$ body weight was exerted on the harness ropes in the second after the triggering of the obstacle. Outcomes with larger integrated forces, and that were not falls, were considered rope assists. Misses resulted when impact with the obstacle did not occur as intended.
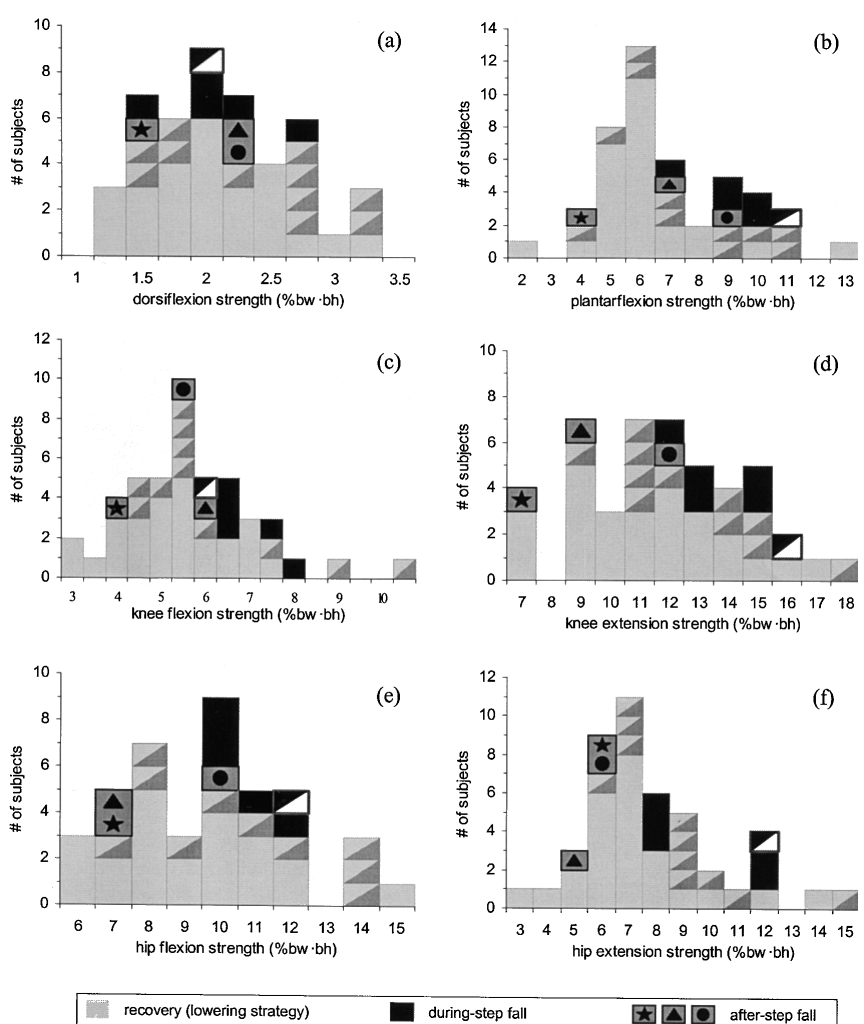

recovery (elevating strategy)

during-step fall during-step fall

国 $\triangle 0$ after-step fall

Figure 1. Histograms illustrating the distribution of the isometric active moment capacities for each group of subjects who successfully recovered or fell after the trip. Distributions are shown for (a) ankle dorsiflexion, (b) ankle plantarflexion, (c) knee flexion, (d) knee extension, (e) hip flexion, and (f) hip extension. Each of the three after-step fallers is indicated by a different symbol. Joint angles corresponding to the displayed strengths are as in Table $1 .(\% \mathrm{bw} \cdot \mathrm{bh})=$ percentage of body weight times body height.
Subjects were also classified according to their recovery strategy. In a lowering strategy, the tripped foot was rapidly lowered on the near side of the obstacle, and the contralateral limb performed the initial recovery step across the obstacle. In an elevating strategy, the tripped foot was immediately lifted across the obstacle to perform the initial recovery step as a continuation of the original step.

Fallers employing a lowering strategy were further categorized based on the timing of their "fall," defined as when $50 \%$ of body weight was supported by the safety harness. ${ }^{17}$ During-step fallers "fell" within 80 milliseconds of recovery step contact with the ground. After-step fallers "fell" at least 470 milliseconds after recovery step contact.

\section{Statistics}

Pearson correlations were computed between the measures of lower extremity strength. Measures judged from these correlations to be related to one another were subjected to

Table 2. Pattern Matrix for the Seven Factors Describing the Lower Extremity Strength Measures

\begin{tabular}{|c|c|c|c|c|c|c|c|c|}
\hline \multirow[b]{2}{*}{ Strength Measure } & \multicolumn{7}{|c|}{ Factor } & \multirow[b]{2}{*}{$r^{2}$} \\
\hline & DF & PF & $\mathrm{KF}$ & $\mathrm{KE}$ & $\mathrm{HF}$ & $\mathrm{HE}$ & ROI & \\
\hline \multicolumn{9}{|l|}{ Ankle dorsiflexion } \\
\hline Isometric & .87 & & & .17 & & & & .90 \\
\hline $30 \%$ s concentric & .90 & & & & & & & .92 \\
\hline $30 \%$ s eccentric & .80 & & .15 & & & & -.20 & .84 \\
\hline Rate of increase & .68 & .35 & & & & & & .84 \\
\hline \multicolumn{9}{|l|}{ Ankle plantarflexion } \\
\hline Isometric & & .86 & & & & & & .90 \\
\hline $30 \%$ s concentric & & .84 & & & & .15 & & .89 \\
\hline $30 \%$ s eccentric & & .87 & & .15 & & & & .84 \\
\hline Rate of increase & .18 & .61 & & & & & .32 & .86 \\
\hline \multicolumn{9}{|l|}{ Knee flexion } \\
\hline Isometric & & & .89 & & & & & .92 \\
\hline $30 \%$ s concentric & & & .93 & & & & & .90 \\
\hline $30 \%$ s eccentric & & & .92 & & & & & .92 \\
\hline Rate of increase & & & .68 & & & & .28 & .83 \\
\hline \multicolumn{9}{|l|}{ Knee extension } \\
\hline Isometric & & .20 & .21 & .63 & .21 & & & .87 \\
\hline $30 \%$ s concentric & & & & .73 & & .18 & & .86 \\
\hline $30 \%$ s eccentric & & & & .94 & & & & .93 \\
\hline Rate of increase & & .36 & .15 & .52 & & & .31 & .84 \\
\hline \multicolumn{9}{|l|}{ Hip flexion } \\
\hline Isometric & .16 & & & & .85 & & & .88 \\
\hline $30 \%$ s concentric & & & & & .84 & & .15 & .91 \\
\hline $30 \%$ s eccentric & & & & & .85 & & & .91 \\
\hline Rate of increase & & & & & .30 & & .65 & .89 \\
\hline \multicolumn{9}{|l|}{ Hip extension } \\
\hline Isometric & & & & .21 & & .71 & & .83 \\
\hline $30 \%$ s concentric & & & & & & .75 & .22 & .92 \\
\hline $30 \%$ s eccentric & & & & & & .89 & & .91 \\
\hline Rate of increase & & & & & .20 & .41 & .58 & .88 \\
\hline
\end{tabular}

Note: Values represent the weighting ( -1 to 1$)$ of each factor in a linear combination describing each measure (e.g. isometric ankle dorsiflexion strength $\approx .87 \mathrm{DF}+$ $.17 \mathrm{KE}$ ). Large weights indicate that a factor and strength measure are strongly related. Weights with magnitudes less than 0.15 are not shown. The communality $\left(r^{2}\right)$ indicates the proportion of variance that is explained by the seven factors. $\mathrm{DF}=$ dorsiflexion; $\mathrm{PF}=$ plantarflexion $; \mathrm{KF}=$ knee flexion; $\mathrm{KE}=$ knee extension; $\mathrm{HF}=$ hip flexion; $\mathrm{HE}=$ hip extension; $\mathrm{ROI}=$ rate of increase. 
a factor analysis. Factors were extracted using principal components analysis, with the number of factors retained in the model selected based on the resulting scree plot. A direct oblimin factor rotation was employed, with $\delta$, the parameter governing factor obliqueness, equal to 0 . Overall flexion, overall extension, and total strength were quantified by extracting the first principal component (the factor explaining the most variance) from the corresponding set of isometric, concentric, and eccentric strength measures at all joints. Sets thus comprised nine flexion, nine extension, and 18 total strength measures, respectively. Factor scores were computed using the regression method.

The Mann-Whitney test was used to compare the strength factor scores of the during-step and after-step fallers with those who successfully recovered from the trip using a similar, lowering strategy. A significance level of .05 was used. Analyses were performed using SPSS 7.0.

\section{RESULTS}

Strength varied greatly across the 63 subjects for whom complete data remained after processing (see Table 1 and Figure 1). Coefficients of variation for isometric active moment capacity ranged from $21 \%$ to $31 \%$ across joints and exertion directions. The different strength measures were highly and significantly $(P<.001)$ correlated for a given exertion direction at a joint. Measured strength was only moderately correlated across joints and exertion directions; correlations between isometric active moment capacities ranged from 0.37 to 0.70 (all $P<.001$ ), with a root-mean-square $r$-value of 0.53 .

Seven common factors were identified as underlying the 24 measures of lower extremity strength (see Table 2). These seven factors explained $88.2 \%$ of the variance in the strength measures. Six unique factors were associated with general strength in flexion and extension at the ankle, knee, and hip. The seventh factor was associated with the ability to generate rapid rates of moment increase. The factors for overall flexion, overall extension, and total strength explained $61 \%, 63 \%$, and $54 \%$ of their respective variances in measured strength, based on a near-equal weighting of all associated strength measures in the factor scores.

Sixty-one subjects were successfully tripped. Fortythree subjects responded using a lowering strategy, 15 responded using an elevating strategy, and three responses were not classifiable as either strategy. ${ }^{17}$ Of those employing a lowering strategy, there were 26 recoveries, five during-step falls, three after-step falls, and nine rope assists. Of those employing an elevating strategy, there were 11 recoveries, one fall, and three rope assists. Seven subjects among the lowering strategy recoveries and one among the elevating strategy recoveries were excluded from further analysis because of incomplete strength data.

During-step fallers were significantly stronger in ankle plantarflexion, knee flexion, overall extension, and total strength than the subjects who successfully recovered from the trip using a comparable, lowering strategy (Table 3). Similarly, the plantarflexion and overall extension strength scores of the elevating-response faller were more than one standard deviation above the average for those who successfully recovered using an elevating strategy. Trends toward greater strength in the during-step and elevatingresponse fallers were seen in almost every factor.

Differences in strength between the after-step fallers and those who successfully recovered using a lowering strategy were not significant $(P>.05$; Table 3$)$. Nevertheless, in all factors except plantarflexion, the after-step fallers exhibited strength that was below the average for those who recovered, with deficits averaging 0.40 in the factor scores. For all three groups of fallers, the individual strength measures exhibited the same trends seen in the factor scores (Figure 1).

Table 3. Comparison of Lower Extremity Strength Factor Scores Between Each Group of Fallers and Those Who Recovered Using a Similar Strategy

\begin{tabular}{|c|c|c|c|c|c|c|c|}
\hline \multirow[b]{3}{*}{ Factor } & \multicolumn{5}{|c|}{ Lowering Strategy } & \multicolumn{2}{|c|}{ Elevating Strategy } \\
\hline & $\begin{array}{l}\text { Recovery } \\
(n=19)\end{array}$ & $\begin{array}{l}\text { During-Step Fall } \\
\qquad(\mathrm{n}=5)\end{array}$ & & $\begin{array}{l}\text { After-Step Fall } \\
\quad(\mathrm{n}=3)\end{array}$ & & $\begin{array}{l}\text { Recovery } \\
(n=10)\end{array}$ & \\
\hline & mea & $\pm S D$ & $P$-Value* & mean $\pm S D$ & $P$-Value* & mean $\pm S D$ & Fall $(n=1)$ \\
\hline DF & $-0.20 \pm 1.17$ & $-0.14 \pm 1.16$ & 945 & $-0.39 \pm 0.81$ & .857 & $0.42 \pm 1.23$ & -0.49 \\
\hline PF & $-0.36 \pm 0.83$ & $0.91 \pm 0.69$ & .009 & $-0.22 \pm 1.46$ & .857 & $0.36 \pm 1.14$ & 2.43 \\
\hline KF & $-0.31 \pm 0.81$ & $0.60 \pm 0.64$ & .044 & $-0.57 \pm 0.27$ & .408 & $0.48 \pm 1.06$ & 0.08 \\
\hline KE & $-0.09 \pm 1.26$ & $0.39 \pm 0.63$ & .208 & $-0.82 \pm 0.91$ & .408 & $0.35 \pm 0.63$ & 0.89 \\
\hline $\mathrm{HF}$ & $-0.40 \pm 1.07$ & $0.28 \pm 0.57$ & 183 & $-0.77 \pm 0.76$ & .718 & $0.50 \pm 0.91$ & 1.19 \\
\hline $\mathrm{HE}$ & $-0.34 \pm 0.90$ & $0.30 \pm 0.90$ & .088 & $-0.72 \pm 0.52$ & .718 & $0.40 \pm 1.09$ & 1.18 \\
\hline $\mathrm{ROI}$ & $0.02 \pm 0.92$ & $-0.37 \pm 0.58$ & .446 & $-0.46 \pm 1.02$ & .586 & $0.20 \pm 1.34$ & 1.07 \\
\hline Flexion & $-0.41 \pm 1.02$ & $0.34 \pm 0.48$ & .053 & $-0.75 \pm 0.58$ & .651 & $0.60 \pm 1.06$ & 0.46 \\
\hline Extension & $-0.37 \pm 1.05$ & $0.71 \pm 0.39$ & .015 & $-0.82 \pm 0.81$ & .586 & $0.51 \pm 1.06$ & 1.81 \\
\hline Total & $-0.41 \pm 1.02$ & $0.56 \pm 0.41$ & .019 & $-0.85 \pm 0.73$ & .651 & $0.59 \pm 1.07$ & 1.22 \\
\hline
\end{tabular}

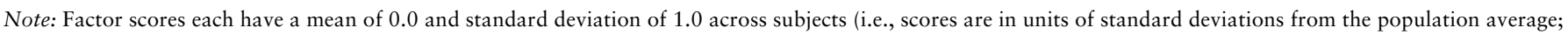
$\mathrm{n}=63$ ). Larger values correspond to greater strength.

* Fall group versus recovery group for the lowering strategy.

$\mathrm{DF}=$ dorsiflexion; $\mathrm{PF}=$ plantarflexion; $\mathrm{KF}=$ knee flexion; $\mathrm{KE}=$ knee extension; $\mathrm{HF}=$ hip flexion; $\mathrm{HE}=\mathrm{hip}$ extension; $\mathrm{ROI}=$ rate of increase. 


\section{DISCUSSION}

The main objective of this study was to determine whether decreased lower extremity strength contributed to the tripinduced falls that occurred by any of three identified mechanisms in a population of healthy older adults. Contrary to expectations, two of three groups of fallers exhibited greater strength than those who recovered. Although contrary to the general and consistent observations of decreased strength in older adults who fall, ${ }^{11-14}$ our results make sense within the present cohort. A primary contributor to the during-step and elevating-response falls was a faster walking speed at the time of the trip than in those who recovered $(1.44 \pm 0.10$ vs $1.13 \pm 0.19$ meters $/ \mathrm{sec}-$ ond). ${ }^{17}$ In older adults, faster walking speeds and longer steps during normal gait are associated with greater plantarflexion and knee extension strength, ${ }^{26-28}$ and these are essentially the factors in which the fallers exhibited greater strength. The correlations between normal walking speed and the factors describing plantarflexion and overall extension strength $(0.29$ and 0.47 , respectively) suggest that the strongest healthy older adults are at an increased risk of falling after a trip because of the faster normal walking speeds that accompany this strength.

After-step falls might reasonably be related to lower extremity weakness, particularly of the hip and knee extensors, because primary factors in the apparent mechanism of these falls were excessive lumbar flexion and buckling of the recovery limb. ${ }^{17}$ Although the results did not provide statistical support for such a relationship, two of three afterstep fallers were among the weakest subjects tested (Figures 1 and 2). In addition, the strength demands on these fallers were likely higher than on those who recovered, because the after-step fallers exhibited a lower hip height, more inclined trunk, and greater head-arm-torso forward angular velocity at recovery step ground contact (mean differences: 3.6\% body height, $19.2^{\circ}$, and $79.1 \%$ second, respectively). ${ }^{17}$ Therefore, we believe that decreased lower extremity strength contributed directly to at least two of the after-step falls. Nevertheless, within the measured ranges of strength, there were no discernible threshold levels below which most subjects had insufficient strength to recover. In most instances, recovery from a trip did not require lower extremity strength exceeding the capacity of the healthy older adults tested, even in the weakest subjects.

The present study has a number of limitations. First, the subjects were a population of healthy, communitydwelling older adults, most less than 80 years old. Therefore, despite ranging widely in strength, they probably represent the strongest older adults. Differing influences of strength on falling might be observed in a weaker population. However, if decreased lower extremity strength contributed to the after-step falls by the present subjects, it should also contribute to these types of falls in weaker populations of older adults. Second, the small number of subjects in each group of fallers allowed only larger influences of strength to be detected; the strengths of the during-step and after-step fallers needed to rank, on average, within the lowest or highest 7.0 and 4.7 scores, respectively, to establish significance. As a result, smaller, but potentially important, influences could remain undetected. We believe that this occurred with the after-step fallers. Finally, common mechanisms of falling from a trip other
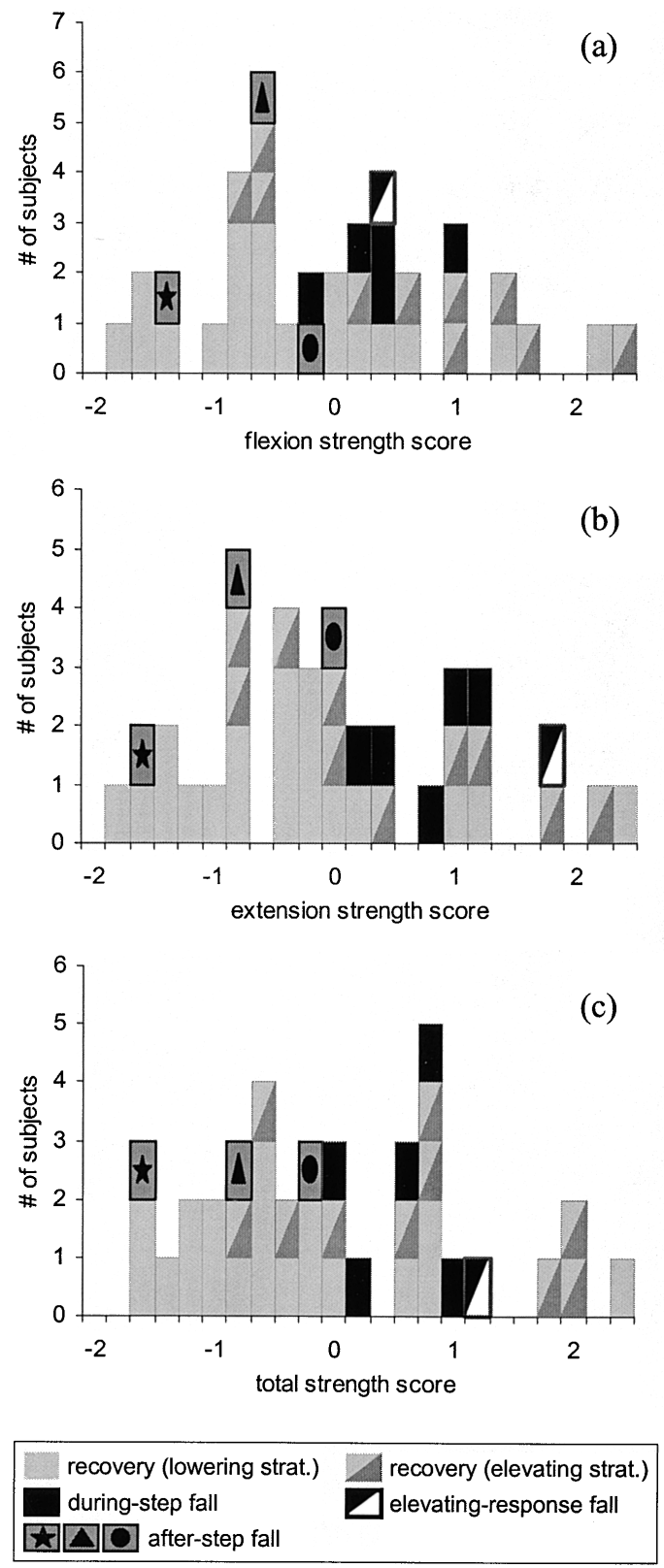

Figure 2. Histograms illustrating the distribution of the factor scores describing (a) overall flexion strength, (b) overall extension strength, and (c) total strength for each group of subjects who successfully recovered or fell after the trip. Each of the three after-step fallers is indicated by the same symbol as in Figure 1. Two of three after-step fallers were among the weakest subjects.

than those observed may exist across the population of older adults, and strength may play a greater role in these unobserved mechanisms. Our conclusions are necessarily limited to the mechanisms observed.

We implicitly assumed that the strength measures employed adequately characterized the functional strength capacity of the older adults tested. For each exertion direction at each joint, strength was essentially associated with a single factor. This indicates that the different conditions tested provided largely redundant information and argues against the need for additional measurements. However, the association of a unique factor with strength in each di- 
rection at each joint indicates that each of these strengths was necessary to fully characterize lower extremity strength. Only $55 \%$ of the total variance in the strength measures could be accounted for by a single factor. The extent to which our quantified factors correspond to functional capabilities during a complex motor task requiring coordinated strength at multiple joints is presently unknown.

In summary, the present results suggest that weak older adults and the strongest older adults may be at increased risk of falling after a trip. High lower extremity strength appears to increase the likelihood of a during-step or elevating-response fall, because of the associated faster walking speeds. Conversely, decreased strength may increase the likelihood of an after-step fall by limiting the ability to execute the required motor response. This suggests that, by working to maintain or increase their lower extremity strength and being aware of the risks of walking quickly, older adults may be able to reduce their likelihood of falling after a trip.

\section{ACKNOWLEDGMENTS}

The authors thank S. Tina Biswas, Rachel A. Brady, and Lesley A. DeBrier for assisting in data collection and processing and Brian L. Sauer for his design and fabrication of the mechanical obstacle.

\section{REFERENCES}

1. Blake AJ, Morgan K, Bendall MJ et al. Falls by elderly people at home: Prevalence and associated factors. Age Ageing 1988;17:365-372

2. Campbell AJ, Borrie MJ, Spears GF. Risk factors for falls in a communitybased prospective study of people 70 years and older. J Gerontol 1989;44: M112-M117.

3. Tinetti ME, Speechley M, Ginter SF. Risk factors for falls among elderly persons living in the community. N Engl J Med 1988;319:1701-1707.

4. Aniansson A, Hedberg M, Henning G-B et al. Muscle morphology, enzymatic activity, and muscle strength in elderly men: A follow-up study. Muscle Nerve 1986;9:585-591.

5. Cahalan TD, Johnson ME, Liu S et al. Quantitative measurements of hip strength in different age groups. Clin Orthop 1989;246:136-145.

6. Larsson L, Grimby G, Karlsson J. Muscle strength and speed of movement in relation to age and muscle morphology. J Appl Physiol 1979;46:451-456.

7. Vandervoort AA, McComas AJ. Contractile changes in opposing muscles of the human ankle joint with aging. J Appl Physiol 1986;61:361-367.

8. Winegard KJ, Hicks AL, Sale DG et al. A 12-year follow-up study of ankle muscle function in older adults. J Gerontol A Biol Sci Med Sci 1996;51A: B202-B207.

9. Häkkinen K, Häkkinen A. Muscle cross-sectional area, force production and relaxation characteristics in women at different ages. Eur J Appl Physiol $1991 ; 62: 410-414$.

10. Thelen DG, Schultz AB, Alexander NB et al. Effects of age on rapid ankle torque development. J Gerontol A Biol Sci Med Sci 1996;51A:M226-M232.

11. Gehlsen GM, Whaley MH. Falls in the elderly: Part II, balance, strength, and flexibility. Arch Phys Med Rehabil 1990;71:739-741.

12. Robbins AS, Rubenstein LZ, Josephson KR et al. Predictors of falls among elderly people: Results of two population-based studies. Arch Intern Med 1989;149:1628-1633.

13. Studenski S, Duncan PW, Chandler J. Postural response and effector factors in persons with unexplained falls: Results and methodologic issues. J Am Geriatr Soc 1991;39:229-234.

14. Whipple RH, Wolfson LI, Amerman PM. The relationship of knee and ankle weakness to falls in nursing home residents: An isokinetic study. J Am Geriatr Soc 1987;35:13-20.

15. Province MA, Hadley EC, Hornbrook MC et al. The effects of exercise on falls in elderly patients: A preplanned meta-analysis of the FICSIT trials. JAMA 1995;273:1341-1347.

16. Pavol MJ, Owings TM, Foley KT et al. The sex and age of older adults influence the outcome of induced trips. J Gerontol A Biol Sci Med Sci 1999;54A: M103-M108.

17. Pavol MJ, Owings TM, Foley KT et al. Mechanisms leading to a fall from an induced trip in healthy older adults. J Gerontol A Biol Sci Med Sci 2001;56A: M428-M437.

18. Eng JJ, Winter DA, Patla AE. Intralimb dynamics simplify reactive control strategies during locomotion. J Biomech 1997;30:581-588.

19. Schillings AM, van Wezel BMH, Mulder TH et al. Muscular responses and movement strategies during stumbling over obstacles. J Neurophysiol 2000; 83:2093-2102.

20. Wojcik LA, Thelen DG, Schultz AB et al. Age and gender differences in peak lower extremity joint torques and ranges of motion used during single-step balance recovery from a forward fall. J Biomech 2001;34:67-73.

21. Schultz, AB. Muscle function and mobility biomechanics in the elderly: An overview of some recent research. J Gerontol A Biol Sci Med Sci 1995; 50A(Special Issue):60-63.

22. Pavol MJ, Grabiner MD. Estimation of Knee and Hip Joint Moments from KIN-COM Forces. Proceedings of the 21st Annual Meeting of the American Society of Biomechanics. Clemson, SC: American Society of Biomechanics, September 24-27, 1997, pp 280-281.

23. Pavol MJ. Biomechanical Determinants of the Outcome of an Induced Trip in Healthy Older Adults [dissertation]. Columbus, OH: The Ohio State University, 1999, pp 167-175.

24. Kannus P, Beynnon B. Peak torque occurrence in the range of motion during isokinetic extension and flexion of the knee. Int J Sports Med 1993;14:422-426

25. Foley KT, Owings TM, Pavol MJ et al. Maximum grip strength is not related to bone mineral density of the proximal femur in older adults. Calcif Tissue Int 1999;64:291-294.

26. Bendall MJ, Bassey EJ, Pearson MB. Factors affecting walking speed of elderly people. Age Ageing 1989;18:327-332.

27. Judge JO, Davis RB III, Õunpuu S. Step length reductions in advanced age: The role of ankle and hip kinetics. J Gerontol A Biol Sci Med Sci 1996;51A: M303-M312.

28. Lord SR, Lloyd DG, Li SK. Sensori-motor function, gait patterns and falls in community-dwelling women. Age Ageing 1996;25:292-299. 